\title{
Nickel ion level in scoliotic patients implanted with nitrogen plasma surface modified nickel-titanium superelastic spinal implant
}

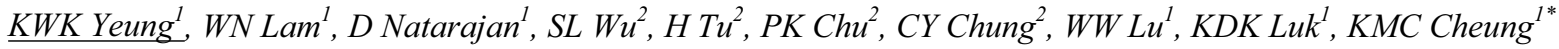 \\ ${ }^{1}$ Division of Spine Surgery, Department of Orthopaedics and Traumatology, The University of Hong Kong, Pokfulam, Hong Kong, \\ China \\ ${ }^{2}$ Department of Physics and Materials Science, City University of Hong Kong, Tat Chee Avenue, Kowloon, Hong Kong, China
}

\section{e-mail:wkkyeung@hku.hk}

Introduction: Nickel-titanium (NiTi) shape memory alloys have been used as surgical implants in orthopaedic procedures. These materials have two distinctive properties, superelasticity and shape memory, that most other current medical metallic materials do not possess. Toxic nickel particulate debris released from these materials, however, remains a major concern particularly pertaining to orthopaedic implants for which fretting is always expected at the implant junction. Metallic debris is suspected to be associated with postoperation complications such as implanted-induced osteolysis, pseudoarthrosis, subacute low-grade implant infection, late operative site pain and abnormal metal concentration in serum. Therefore, an advanced surface technology using plasma immersion ion implantation (PIII) has been developed to address this issue. This paper describes serum nickel ion level monitoring of patients with scoliosis operated upon using nitrogen plasma implanted NiTi spinal rods compared with standard titanium alloy rods in a randomized human clinical trial.

Methods: NiTi spinal rods with $50.8 \% \mathrm{Ni}$ were treated by nitrogen PIII at $40 \mathrm{kV}$ with $100 \mathrm{~Hz}$. Seventeen patients with spinal scoliosis were surgically treated by either Ti alloy rods or nitrogen plasma implanted NiTi rods. The mean age at operation was 16.6 years. The mean number of treated spinal segments was 9. Blood samples were collected as baseline before surgery and up to 1 year post-surgery. Nickel levels were assayed by inductively coupled plasma mass spectrometry. Twelve patients $(7 \mathrm{M}$ and $5 \mathrm{~F}, 6=\mathrm{NiTi}$ and $6=\mathrm{Ti})$ were followed up for 6 months, and five patients $(2 \mathrm{M}$ and $3 \mathrm{~F}$, 2=NiTi and 3=Ti) had completed 1-year follow-up.

Results: From Figures 1 and 2, in all cases, patients who had NiTi rods implanted had a 2.5 times increase in the Ni level at Day 1 after surgery as compared with before surgery. However, all were within recommended safe limits. The Ni levels returned to baseline in all subjects between 7 days to 1 month post-surgery. No clinical signs and symptoms of Ni allergy or toxicity was observed. The patients who had Ti alloy rods implanted remained no change.
Discussion: This study has successfully demonstrated that the nitrogen plasma treated nickel titanium spinal rods are safe to the patients while undergoing scoliosis correction surgery. The increased nickel level in the patients with NiTi rods may attribute to the unprotected ends. Due to the surgical maneuver, the two ends of the rods were cut to fit the operated length of patients. Therefore, the untreated ends were exposed and contacted with the surrounding human tissues and bloods. However, the nickel levels gradually returned to the baseline. We believe that the unprotected ends had been protected by the newly formed oxide layer after a period of operation.

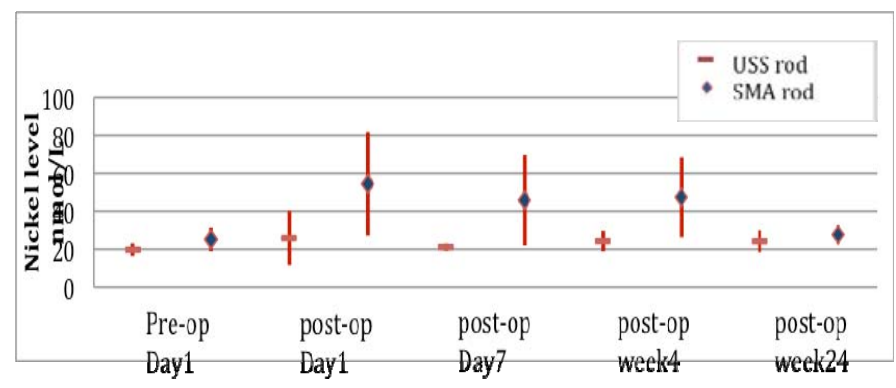

Figure 1 Serum nickel level from pre-op Day 1 to post-op 24 weeks of the patients using SMA and USS rod.

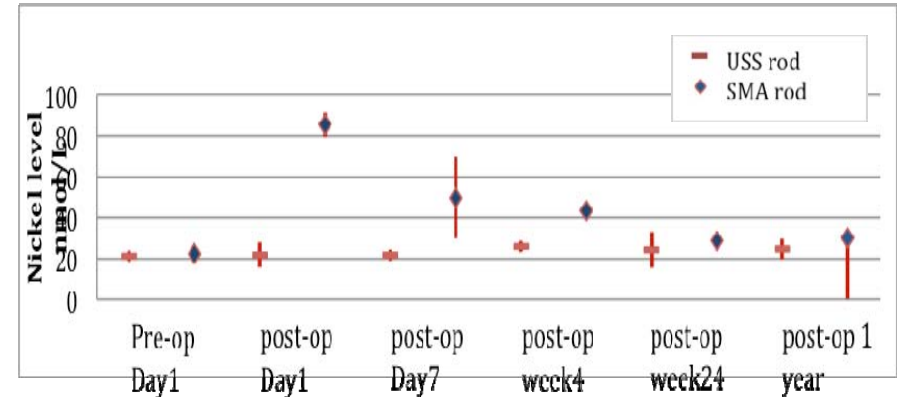

Figure 2 Serum nickel level from pre-op Day 1 to post-op 1 year of the patients using SMA and USS rod. 